\title{
Balkanologie
}

Balkanologie Revue d'études pluridisciplinaires

Vol. I, n 1 | 1997

Volume I Numéro 1

\section{Politique panroumaine et identité moldove}

\section{Charles King}

Traducteur : Patrick Michels et Suzana Đurić

\section{(2) OpenEdition}

\section{Journals}

Édition électronique

URL : http://journals.openedition.org/balkanologie/195

DOI : $10.4000 /$ balkanologie. 195

ISSN : 1965-0582

Éditeur

Association française d'études sur les Balkans (Afebalk)

Édition imprimée

Date de publication : 1 juillet 1997

ISSN : 1279-7952

\section{Référence électronique}

Charles King, «Politique panroumaine et identité moldove », Balkanologie [En ligne], Vol. I, n 1 | 1997,

mis en ligne le 02 juin 2008, consulté le 17 décembre 2020. URL : http://journals.openedition.org/

balkanologie/195; DOI : https://doi.org/10.4000/balkanologie.195

Ce document a été généré automatiquement le 17 décembre 2020.

(c) Tous droits réservés 


\title{
Politique panroumaine et identité moldove
}

\author{
Charles King \\ Traduction : Patrick Michels et Suzana Đurić
}

\section{NOTE DE L'ÉDITEUR}

Cet article est paru dans Slavic Review (New York), 53 (2), summer 1994, pp. 345-368. Il a été traduit de l'anglais avec l'aimable autorisation de l'éditeur (the American Association for the Advancement of Slavic Studies - AAASS) et de l'auteur par Patrick Michels et Suzana Djuric.

L'auteur tient à remercier Igor Botan, Mihai Gribincea, Igor Munteanu et Valeriu Opinca pour leur aide appréciable, ainsi que les membres anonymes du comité de lecture [de la S.R.] pour leurs commentaires incisifs. Les recherches pour cet article ont pu être menées grâce aux aides du Marshall Aid Commemoration Commission, du Social Science Research Council et de St. Anthony's College, Oxford.

Ce n'est qu'au début du XVIII ${ }^{\text {ème }}$ siècle, alors que le prince moldove Dimitrie Cantemir était la vedette des salons de Berlin à St Petersbourg, que l'existence d'un État moldove ${ }^{1}$ fut aussi largement reconnue par la communauté internationale. La Moldovie quitta l'Union soviétique le 27 août 1991, mais son admission aux Nations unies l'année suivante souleva l'angoissante question de l'orientation future de la république. La question de savoir si la Moldovie doit renforcer ses liens avec ses anciennes républiques-soeurs dans la Communauté d'États Indépendants (CEI) ou s'orienter vers la réintégration à la Roumanie, le pays auquel une grande partie de son territoire appartenait avant 1940, continue de diviser les acteurs politiques de cette république. Bien que les nombreux partis politiques du pays aient aussi d'autres problèmes urgents à résoudre, tels que la restructuration économique et la réforme constitutionnelle, ils ont été contraints de prendre partie dans l'éternel débat entre les factions " prounification » et « pro-indépendance » au sein de l'élite politique et culturelle. 
2 D'ailleurs, d'importantes questions sur la signification de la "moldovité" elle-même ont inévitablement pris place dans le débat public. Pour beaucoup d'écrivains et autres intellectuels de Moldovie, l'indépendance moldove constitue le premier pas vers la réunion à la mère-patrie roumaine. Pour ces groupes - que j'appelle "panroumains" "moldove" ne devrait pas désigner autre chose qu'une identité régionale dans une "Grande Roumanie" reconstituée. À l'autre extrême, de puissants regroupements politiques soutiennent que, bien que les Moldoves soient liés aux Roumains par une langue et une culture communes, l'histoire a «condamné [la Moldovie] à former un État » et ses citoyens ne doivent pas attendre l'unification comme « une manne tombée $\mathrm{du}$ ciel $\aleph^{2}$. Pour les plus radicaux de ces groupes - familièrement appelés les "moldovistes" - les Moldoves devraient rejeter complètement l'ethnonyme "Roumain" et poursuivre la construction d'une république moldove indépendante au sein de la CEI. Tout discours sur l'intégration panroumaine, que ce soit dans un sens culturel ou politique, utilise simplement de "hauts idéaux" afin de "masquer des intérêts personnels dans le démembrement territorial de la république $»^{3}$. Depuis 1990, l'existence de républiques sécessionnistes dans le sud et l'est de la Moldovie (Gagaouzie et Transnistrie) a conféré une urgence particulière au problème de l'identité nationale et forme un point d'interrogation quant à la future configuration territoriale de l'État.

Bien que la construction de nouvelles identités post-soviétiques soit devenue une caractéristique commune de la vie politique dans les États successeurs, la saillance de la politique identitaire est particulièrement accentuée dans le cas moldove ${ }^{4}$. Les principaux journaux réservent un espace important aux discussions sur la linguistique, l'ethnographie et l'histoire médiévale; la publication de lettres d'organisations culturelles et professionnelles accusant le gouvernement moldove de trahir l'idéal panroumain est fréquente; et les politiciens moldoves eux-mêmes ont présenté des arguments historiques ou littéraires pour défendre leurs propres conceptions de la moldovité. Néanmoins, insister sur la question identitaire représente plus qu'une rhétorique politique. En fait, étant donné les profondes divisions de la société moldove, avec la grande partie de son intelligentsia panroumaine déniant la légitimité de l'État lui-même, des disputes apparemment ésotériques à propos de l'ethnographie ou de la linguistique ont nécessairement de réelles conséquences politiques, particulièrement en ce qui concerne les partis politiques, le conflit ethno-territorial et les relations avec la Roumanie. Alors que les écrivains, les artistes et les universitaires sont normalement perçus comme les gardiens du nationalisme officiel, la Moldovie fournit un cas fascinant sur la gravité de la politique identitaire lorsque la représentation que professent les politiciens diverge radicalement de celle que préconisent les intellectuels.

\section{Les antécédents de la Moldovie}

4 Selon une légende populaire, le nom "Moldova" provient des aventures de Dragos, prince transylvanien semi mythique qui chassait dans les régions à l'est des montagnes des Carpathes en 1359. Lorsque la partie de chasse de Dragos tomba sur un boeuf sauvage près d'un torrent, son chien de chasse préféré, Molda, se jeta sur l'animal et se noya dans la lutte qui s'ensuivit. En mémoire de l'événement, Dragos appela la rivière "Molda" et prit comme sceau la tête de boeuf ; après avoir exploré la région au-delà des Carpathes, Dragos se rappella encore du chien et donna le nom de "Moldova" à la principauté qu'il établit dans la région ${ }^{5}$. Suite à des débuts si peu propices, la 
principauté de Moldovie émergea au XVème siècle comme une des puissances majeures de l'Europe orientale. Sous le règne du puissant prince Stefan le Grand (1457-1504), la Moldovie atteignit l'apogée de sa puissance politique et militaire. S'étendant de l'est des Carpathes au Dniestr et à la Mer Noire, la Moldovie était très convoitée par les Hongrois, les Polonais, les Tatars, ainsi que par les Turcs. Stefan s'efforça de les opposer les uns aux autres durant une grande partie de son règne. Avec les successeurs de Stefan, la principauté capitula finalement devant l'avancée des Ottomans vers le nord, et elle devint un État vassal de la Sublime Porte en 1538.

Suite au Traité de Bucarest de 1812, ayant temporairement mit fin aux hostilités entre les empires russe et ottoman, le Tsar Alexandre $\mathrm{I}^{\mathrm{er}}$ étendit le contrôle russe sur la moitié orientale de la Principauté de Moldovie, la région entre le Prout et le Dniestr, à laquelle les cartographes du Tsar donnèrent le nom de "Bessarabie"6. En 1859, la moitié occidentale de Moldovie s'unit à la principauté voisine du sud-ouest, la Valachie, et prit plus tard le nom de "Roumanie", tandis que la Bessarabie était laissée en dehors du mouvement pour l'unification panroumaine. De la fin de la guerre de Crimée au Congrès de Berlin (1856-1878), trois districts des rives de la Mer Noire retournèrent dans les principautés roumaines, mais, mis à part ces deux décennies, la totalité de la Bessarabie demeura une guberniia arriérée sur la frange occidentale de l'empire russe.

Après la révolution de 1905 à St Petersbourg, beaucoup d'intellectuels bessarabiens virent dans l'agitation en Russie une opportunité d'étendre le nationalisme panroumain entre les fleuves. Lors de la révolution de février, les nombreux groupes de discussion politique et les cercles littéraires formés après 1905 avaient formé le Parti National moldove, organisation qui eut un rôle décisif dans la proclamation d'une "République Moldove de Bessarabie" indépendante en janvier 1918. La politique dans l'éphémère république indépendante produisit autant de factions que dans les autres parties de l'empire, des mouvements "pan», dont le but était l'unification avec une mère-patrie ethnique, aux élites russifiées demandant le retour du Tsar, en passant par les socialistes engagés dans la réforme de la terre et l'émancipation politique. Finalement, ce fut la Roumanie qui détermina le résultat de cette joute politique interne. La chute du Tsar offrit à la Roumanie la chance de retrouver les territoires perdus en 1812, puis en 1878. Sous le prétexte de restaurer l'ordre le long de ses frontières orientales, les troupes roumaines franchirent le Prout et occupèrent la Bessarabie au début de 1918, un mouvement bien accueilli par les panroumains de Chisinau, mais critiqué par les groupes ayant peu d'affinités pour la monarchie roumaine. Face au choix entre les bolcheviques et Bucarest, l'Assemblée Nationale de Bessarabie, ou Sfatul Tarii, vota pour l'unification avec le Royaume de Roumanie en mars 1918 et en décembre rejoignit plusieurs territoires de l'ex-Autriche-Hongrie au sein des nouvelles frontières de la Roumanie aggrandie ${ }^{7}$.

7 Les leaders politiques bessarabiens, nombre d'entre eux impliqués dans le mouvement socialiste en Russie, espéraient pouvoir jouer un rôle progressiste dans la Grande Roumanie. Toutefois, l'euphorie de l'unification panroumaine s'éteignit relativement rapidement. Les Bessarabiens furent traités comme des cousins pauvres par leurs frères de jadis à Bucarest, et les demandes d'une réforme électorale radicale tombèrent dans les oreilles sourdes du palais de Bucarest ${ }^{8}$. La Bessarabie demeura une partie sousdéveloppée de la grande Roumanie, tout comme l'était la "Sibérie occidentale" dans l'Empire russe. Plus significativement, l'enthousiasme panroumain de Bucarest n'avait reçu aucun écho dans les villages bessarabiens, dans lesquels vivaient $87 \%$ de la 
population totale de Bessarabie et $93 \%$ des locuteurs roumains de la région ${ }^{9}$. Les voyageurs occidentaux notèrent l'utilisation continuelle de l'ethnonyme "Moldove"10, et les batailles majeures furent menées autour de réformes telles que l'alphabet latin, le calendrier grégorien et de nouvelles heures d'ouvertures des magasins ${ }^{11}$.

8 La Bessarabie demeura une pomme de discorde entre Bucarest et Moscou après 1918, puisque son incorporation dans la Roumanie ne fut jamais tout à fait reconnue ni par les puissances occidentales, ni par les bolcheviques. À la suite d'une rupture dans les négociations roumano-soviétique à propos de la Bessarabie en 1924, les Soviétiques établirent une "République Socialiste Soviétique Autonome Moldove" (RSSAM) sur la rive orientale du Dniestr en Ukraine. La RSSAM servit de tête-de-pont à l'influence soviétique dans l'inter-fleuve. D'après les propagandistes soviétiques, les frontières occidentales de jure de la RSSAM (et par conséquent de l'Union Soviétique) se trouvaient le long du Prout, donc incluaient toute la "Bessarabie occupée" dans la république autonome. L'existence de la RSSAM fut présentée par les Soviétiques comme une évidence du fait que, loin de constituer une partie d'une unique nation panroumaine, les Moldoves et les Roumains formaient en réalité deux groupes ethniques totalement distincts, parlant des langues romanes orientales distinctes. Cependant, les Moldoves / Roumains représentaient, tout au plus, moins d'un tiers de la population de la $\operatorname{RSSAM}^{12}$.

9 La signature du pacte Molotov-Ribbentrop en août 1939 permit à l'Union Soviétique d'accroître sa pression sur la Roumanie pour le retour de la Bessarabie sans crainte d'interférence allemande. En juin 1940, l'Union Soviétique annexa de force la Bessarabie et fusionna des parties de l'interfleuve avec une partie de la RSSAM, la bande de territoire à l'est du Dniestr connu aujourd'hui sous le nom de "Transnistria" (en roumain) ou "Pridnestrov'e" (en russe). Dans la nouvelle République Socialiste Soviétique Moldove (RSSM), la politique soviétique se concentra sur l'établissement de barrières entre les Moldoves et les Roumains à l'ouest du Prout. Les famines et les déportations forcées dans les années 1940, la relocalisation "volontaire" des familles moldoves dans les années 1950 et 1960, et l'immigration de Russes et d'Ukrainiens modifia le paysage démographique et rendit littéralement l'ancienne Bessarabie plus "soviétique" que roumaine. L'alphabet cyrillique fut réintroduit en 1941, les historiens découvrirent des liens séculaires entre les Moldoves et les autres nations de l'URSS, et les linguistes soulignèrent les différences fondamentales entre les deux principales langues romanes orientales, le moldove et le roumain - point de vue rejeté par les universitaires hors $\mathrm{d}^{\prime} \mathrm{URSS}^{13}$.

\section{Les débats autour de la langue moldove}

10 La question d'une langue moldove distincte fournit l'arme principale de l'arsenal des "groupes informels" moldoves apparus en été 1988. Les Soviétiques avaient pendant longtemps considéré le critère linguistique comme fondamental dans l'identité nationale et, aussi longtemps que la notion d'une langue moldove distincte put être soutenue, l'idée d'une nation moldove non-roumaine demeura une proposition viable. Les écrivains, artistes, historiens et linguistes moldoves - regroupés dans la principale organisation informelle moldove, le Front Populaire de Moldovie - demandaient le rejet de l'alphabet cyrillique en faveur de l'alphabet latin, la reconnaissance officielle de l'unité des langues roumaine et moldove, et la déclaration du roumain comme langue d'État de la RSSM. À la suite de l'exemple des républiques baltes et du Tadjikistan, le 
Soviet Suprême moldove adopta, le 31 août 1989, trois nouvelles lois sur la langue déclarant le moldove langue d'État de la république, "utilisée dans la vie politique, économique, sociale et culturelle et [écrite dans] l'alphabet latin ». Les lois satisfirent quasiment toutes les demandes du Front Populaire, bien que la seule référence manifeste à l'unité des langues moldove et roumaine fût l'admission, plutôt convolutée, du fait que les autorités "[tenaient] comptent de l'identité linguistique moldovoroumaine réellement existante $»^{14}$.

11 Les nouvelles lois sur la langue représentaient la première étape dans la chute du Parti communiste moldove et le premier pas vers l'indépendance moldove hors de l'Union Soviétique. En adoptant les lois malgré l'opposition officielle du parti communiste, le Soviet Suprême Moldove affirmait son pouvoir en tant que véritable corps législatif, remettant ainsi en cause son précédent rôle de courroie de transmission des directives du parti communiste. Le président, Mircea Snegur, fit adopter d'autres mesures législatives significatives par l'intermédiaire du Soviet Suprême moldove. À la fin de l'année 1990, la Moldovie déclara sa souveraineté au sein de l'Union Soviétique et adopta le drapeau tricolore roumain, avec le blason au sceau de tête de boeuf de Dragos et de Stefan le Grand, comme drapeau national. En août 1991, le gouvernement affirma sa volonté d'indépendance, d'abord en refusant de signer le traité d'union proposé et, ensuite, en publiant une condamnation sans équivoque des putschistes à Moscou. Une semaine après l'échec du coup d'état, le parlement moldove déclara la création " d'un État souverain et indépendant, libre de décider du présent et du futur de la patrie sans aucune interférence étrangère $»^{15}$, et Mircea Snegur devint le premier Président élu par le peuple de la Moldovie indépendante à la fin de l'année.

12 À la fin des années 1980, la question de la langue représentait un point sur lequel les intellectuels et des membres de l'élite politique moldove pouvaient se mettre d'accord. Impatients d'affirmer leur pouvoir par rapport au centre soviétique, aussi bien que par rapport au leadership du parti communiste local russifié, les acteurs poliques moldoves / roumains formèrent un front uni avec les intellectuels moldoves / roumains ${ }^{16}$. Leur intérêt mutuel était clair : les écrivains, les artistes et les historiens moldoves espéraient susciter une renaissance de la culture nationale moldove (lire: roumaine), un but pour lequel beaucoup travaillaient depuis les années 1970, tandis que les élites politiques locales espéraient pouvoir utiliser le mouvement national comme un moyen d'obtenir de plus grandes concessions de la part du centre et d'évincer le leadership brejnévien du Parti communiste moldove. Semion Grossu, le premiersecrétaire du parti local, était en poste depuis 1980 et se distinguait par le fait qu'il était le dernier premier-secrétaire républicain à avoir été désigné sous Brejnev et le dernier à avoir été remplacé sous Gorbatchev. Son style personnel et son manque de diplomatie lors des négociations avec le Front Populaire représentaient une continuelle source d'embarras pour Snegur, Nicolae Tiu, Andrei Sangheli et d'autres jeunes membres du Politburo moldove. En août 1989, lorsque la notion d'une distinction linguistique entre le moldove et le roumain était critiquée, Gossu affirmait encore que les deux langues étaient totalement distinctes et que l'alphabet latin était inapte à retranscrire la langue parlée. Pour le personnel du parti plus sagace, tel que Snegur, Grossu était un repoussoir bien utile. Comparé au premier-secrétaire non réformateur, pratiquement tous les membres du Politburo moldove pouvaient se présenter comme des pragmatiques, attachés au renouvellement de la culture nationale moldove / roumaine au sein d'une Union Soviétique refaçonnée. 


\section{Structuration politique autour de la question identitaire}

13 national moldove. Pour de nombreux intellectuels moldoves / roumains, l'adoption des lois sur la langue représentait une affirmation historique de l'“identité réelle" de la majorité ethnique de la Moldovie. Selon les termes du Front Populaire, «le nom historique de notre peuple, que nous avons porté depuis des siècles - un droit dont les chroniques et les manuscrits, les documents historiques des périodes moderne et contemporaine, et les classiques du marxisme-léninisme témoignent - est roumain et notre langue s'appelle la langue roumaine ${ }^{17}$. À son second congrès, en juin 1990, le Front Populaire se déclara en opposition au gouvernement, qui, selon lui, était trop lent à faire sortir la Moldovie de l'URSS, et appela ouvertement à l'unification avec la Roumanie. Adopter une telle position représentait, pour le leadership du Front Populaire, une épreuve décisive: une fois que l'anti-communisme et l'intégration nationale étaient déclarés buts ultimes de l'organisation, il serait aisé de séparer les fervents panroumains des communisants ${ }^{18}$. Lors de son troisième congrès, en février 1992, le Front se transforma d'un "mouvement de masse" en un parti politique (le Front Populaire Chrétien Démocrate) et inclut un engagement évident pour l'unification moldovo-roumaine dans ses statuts: «l'évolution naturelle ces dernières années du mouvement pour la libération nationale ne peut culminer qu'en embrassant l'idéal de l'unité nationale et la réintégration à l'État roumain unitaire. (...) Le Front Populaire Chrétien Démocrate maintient sa position de mouvement national et unioniste, dont l'objectif principal est la réintégration à l'État roumain unitaire $»^{19}$. Comme pour ne pas ajouter à la légitimité de l'existence d'un État moldove distinct, le Front refaçonné a même rejeté le nom de "République de Moldovie" en faveur de "Bessarabie".

Bien que la radicalisation du Front Populaire réalisait la finalité d'obliger les politiciens moldoves à prendre position pour ou contre l'unification avec la Roumanie, elle affaiblissait aussi sérieusement la force numérique de l'organisation elle-même. Le vaste réseau de groupes locaux qui lui avait permit de s'organiser si efficacement en 1989 diminua rapidement une fois que l'unification avec la Roumanie se révéla être la finalité ultime du Front $^{20}$. De plus, avec la défection de membres du Front et de parlementaires au profit des partis politiques nouvellement créés, de même que le départ de son président et de dirigeants en vue pour la Roumanie ${ }^{21}$, l'organisation se scinda en deux groupes : le déjà-existant Front Populaire Chrétien Démocrate et le Congrès de l'Intelligentsia, formé en avril 1993. Comptant parmi ses membres quelques-uns des anciens dirigeants les plus respectés, le Congrès représentait la partie la moins radicale de l'unionisme en Moldovie: bien qu'en faveur de relations rapprochées avec la Roumanie, le Congrès tempérait son unionisme par des appels à la "reconciliation nationale" et, en ce qui concerne le présent, la poursuite de l'existence d'un État moldove indépendant. Ses statuts mentionnent seulement une «intégration économique et spirituelle progressive à la Roumanie » plutôt que l'unification politique immédiate prônée par le Front ${ }^{22}$.

Une autre conséquence du militantisme du Front fut une radicalisation pendante des groupes en faveur de l'indépendance moldove. Le premier de ces groupes fut le Parti Agrarien Démocrate (formé en novembre 1991), le leader de la plus large faction aussi bien dans le Parlement soviétique que dans le Parlement post-soviétique. Les Agrariens, principalement composés de membres de l'ancienne nomenklatura agricole, ont 
continuellement mis l'accent sur le fait que la Moldovie ne devait devenir ni « une province, ni une guberniia d'un autre pays ${ }^{23}$. Les plus radicaux des Agrariens, la faction réunie autour du journal Viata Satului (La vie du village), de même que leurs alliés parmi les communisants du Parti Républicain, ont été jusqu'à rejeter aussi bien l'ethnonyme que le glottonyme "roumain", soutenant l'ancienne version soviétique que les Moldoves étaient ethniquement distincts des Roumains à l'ouest du Prout. De telles affirmations servent l'intérêt des Agrariens en général : occupant les postes les plus puissants dans l'économie essentiellement agraire de Moldovie, ces présidents de fermes collectives, ces managers agro-industriels et ces dirigeants de gouvernements locaux ont compris que se rapprocher de la Roumanie pouvait compromettre leurs positions au pouvoir ${ }^{24}$. De plus, du fait que les échanges agricoles et commerciaux moldoves sont toujours principalement orientés vers les anciennes républiques soviétiques, les Agrariens ne se pressèrent pas pour rompre des liens essentiels en se précipitant dans les bras des Roumains ${ }^{25}$.

16 En dépit de l'affaiblissement du soutien au Front Populaire, aussi bien au Parlement qu'à la campagne, les panroumains purent, en 1993, démontrer une dernière fois leur force à propos de la participation de la Moldovie à la CEI. Au milieu de l'année 1993, le Parlement n'avait toujours pas ratifié la déclaration d'Alma Ata de 1991, et Moscou accentua ses pressions sur la république pour qu'elle prenne une décision ferme sur l'adhésion ou la menaçait de restrictions commerciales punitives. Lorsque l'adhésion à la CEI fut finalement placée sur l'agenda politique, elle provoqua la confrontation la plus signicative entre les panroumains et les forces indépendantistes depuis la déclaration d'indépendance. Alors que la Roumanie s'orientait vers l'adhésion au Conseil de l'Europe en été 1993, de nombreux parlementaires moldoves perçurent le vote sur la ratification de l'adhésion à la CEI comme un choix entre l'“ouest" et l'“est", c'est-à-dire un développement orienté soit vers la Roumanie et l'Europe, soit vers la Russie et les anciennes Républiques soviétiques. Ainsi, les députés panroumains déclarèrent que ratifier l'accord d'Alma Ata revenait à "légitimer la politique expansionniste de la Russie dans cette région, saper le mouvement de renaissance nationale [et] blesser notre dignité nationale ${ }^{26}$.

17 Malgré le soutien du président Snegur à l'adhésion à la CEI, le vote échoua par manque de marge de manoeuvre. Sur plus de 300 députés inscrits, 162 votèrent en faveur de l'adhésion et 22 contre, c'est-à-dire légèrement en-dessous de la majorité absolue requise pour l'adoption de la loi. De fait, seulement 188 députés prirent part au vote, tandis que presque tous les représentants de la région séparatiste de Transnistrie boycottèrent, comme d'habitude, la session parlementaire, et 90 députés unionistes (menés par le Front, le Congrès de l'Intelligentsia et leurs alliés parlementaires) quittèrent le Parlement pour protester contre la procédure de vote ${ }^{27}$. La défection d'Agrariens vers le camp anti-CEI, consécutive aux avertissements des unionistes affirmant que l'histoire jugerait sévèrement ceux qui auront voté pour la perpétuation de la division de la nation roumaine, contribua aussi à l'échec de la motion. Bien que le vote représentât un succès certain pour les panroumains, il signifiait leur chant du cygne. Le fait que la Moldovie ait quitté la CEI est l'illustration de l'immense pouvoir qu'exerça le bloc minoritaire panroumain au parlement, une nouvelle version du Soviet Suprême élu en 1990, ce qui accéléra le mouvement pour la création d'une Assemblée post-soviétique réduite. La présidence du Parlement ignora le vote de non-ratification, 
approuva la signature moldove du traité de la CEI sur l'union économique et annonça de nouvelles élections parlementaires pour le 27 février.

Lors de la campagne électorale, plusieurs alliances politiques tentèrent de minimiser la question identitaire et de porter l'attention sur les problèmes concrets de la privatisation, de la redistribution des terres et de la réforme constitutionnelle. Notamment, les trois principaux groupes réformistes - le Parti Social Démocrate, le Parti Travailliste Démocratique et le Parti de la Réforme - demandèrent aux électeurs, indépendamment de leur opinion sur la question identitaire, de faire front commun sur le problème du dépérissement de l'économie moldove ${ }^{28}$. Quoi qu'il en soit, un congrès spécial formé par le gouvernement moldove, le principal événement politique de la campagne, soutint que l'identité nationale demeurerait le principal cheval de bataille de la campagne électorale. Le congrès Chez nous - la République de Moldovie se tint à Chisinau le 5 février sous l'égide de l'Alliance Civique moldove, une coalition de partis constituée des forces les plus opposées aux idées panroumaines ${ }^{29}$. Les discours de l'écrivain moldove Ion Druta et du leader agrarien démocrate Dumitru Motpan rejetaient l'idée d'unification avec la Roumanie et soulignaient la nécessité de consolider l'indépendance et l'intégrité territoriale moldoves ${ }^{30}$.

L'allocution aux mots choisis du Président Snegur reçut toutefois la plus grande attention et marqua une rupture importante dans le traitement présidentiel de l'identité nationale moldove. Par le passé, Snegur s'ingéniait à se tenir à distance respectable du "moldovisme" des plus radicaux agrariens démocrates, qui était une perception de la distinction moldove-roumaine semblable à la politique soviétique discréditée en 1989. Bien que déniant la possibilité d'une unification politique, Snegur approuva au préalable l'héritage roumain de l'État moldove et le besoin d'intégration culturelle et économique avec Bucarest. Dans son discours «Chez nous », les propos du Président étaient, toutefois, sans équivoque. Snegur dénonça le panroumanisme comme une «trahison » et accusa les écrivains et les historiens moldoves de mettre en doute la « légitimité et les fondements historiques de notre droit à former un État, de nous appeller le peuple moldove». Dans des termes sans équivoques, il souligna l'existence d'un ethnos moldove distinct comme fondement de l'État moldove et, dans un style rappelant le précédent premier-secrétaire Grossu, il évita de prendre position sur la question de l'existence d'une langue moldove distincte :

Il y a eu beaucoup d'agitation à propos de la langue parlée par les Moldoves en République de Moldovie. Bien entendu, nous partageons la même langue avec nos frères de Roumanie. Mais, pareillement, personne ne peut dénier l'existence de certaines nuances [dans la langue moldove] (...) D'après moi, en tant que locuteur parmi d'autres de cette langue, nous ne pouvons remettre en cause le fait que nos frères et nos soeurs parlent lègèrement différemment de nous. L'acceptation de cette différence a été caractéristique tout au long de l'histoire et je ne vois pas pourquoi nous faisons tout ce que nous pouvons pour l'oublier maintenant.

La version imprimée du discours incluait un nombre important de notes de bas de page faisant référence à des travaux historiques et littéraires roumains bien connus dans lesquels le terme "Moldove" était utilisé pour décrire la majorité ethnique de la région du Prout-Dniestr. Contrairement à ses prises de position antérieures, Snegur désigna aussi la république moldove de 1917-1918 comme le précurseur logique de la république de Moldovie :

En 1917, suite à l'écroulement de l'empire tsariste, la constitution d'une République Démocratique Moldove fut déclarée le 2 décembre, et le 24 janvier 1918 la République moldove indépendante fut proclamée. En conséquence, ce qui arriva fin 
août 1991, après le putsch dans l'ancien empire soviétique, c'est-à-dire la décision (...) du Parlement de proclamer la Moldovie État indépendant et souverain, peut et doit être considéré comme la satisfaction des désirs inaltérables du peuple de perpétuer la tradition des Moldoves en tant qu'État-nation... ${ }^{31}$

Évidemment, le discours fut immédiatement condamné par les intellectuels moldoves influents - les représentants de l'Union des Écrivains, de l'Institut de Linguistique, de l'Institut d'Histoire, de l'Université et d'autres institutions - comme un affront à la véritable identité de la majorité ethnique de la République et comme une tentative de perpétuer « une invention du régime communiste » en érigeant une «barrière contre l'autentique culture roumaine $»^{32}$. Les historiens, en particulier, furent outragés de l'utilisation par Snegur de documents historiques pour légitimer la division de la nation roumaine. Une lettre ouverte au président signée par 43 universitaires influents affirmait que :

le glottonyme "langue moldove" ne peut avoir aucune confirmation d'aucune sorte. Il est vrai que la même chose peut être dite à propos de l'“État moldove", et nous aimerions croire que c'est la raison pour laquelle vous avez décidé de nous convaincre que nous somme Moldoves. Il n'y avait aucun besoin de faire une chose pareille. Nous savons très bien que nous sommes Moldoves, (...) tout comme les Italiens sont Milanais, Vénitiens, Piedmontais (...).

Ainsi, il n'est pas difficile de comprendre que le nom "Moldove" ne désigne pas un peuple [popor] en tant que tel, mais bien plutôt une partie de la nation roumaine [neamului romanesc]. Il provient du nom topographique Moldova et est, par conséquent, essentiellement géographique et non ethnique, tout comme les noms des Roumains vivant dans d'autres parties du territoire national. En d'autres mots, Moldoves en vertu de la région où l'on est né, en tant que partie intégrante du peuple roumain, nous sommes en même temps Roumains, que ça nous plaise ou non. ${ }^{33}$

L'adoption par Snegur de la conception "moldoviste" de l'identité nationale aida certainement les Agrariens Démocrates lors des élections de février 1994. La perte de soutien du Front après 1990 avait déjà indiqué que les idées panroumaines ne recevaient que peu d'échos et l'adoption par Snegur d'une rhétorique plus antiunioniste durant la campagne électorale contribua à sceller le sort politique du Front. Les Agrariens émergèrent avec plus de $43 \%$ des voix et une majorité absolue de sièges dans le nouveau Parlement de 104 membres, tandis que les panroumains - divisés entre le Front et le Congrès de l'Intelligentsia, plus pragmatique - n'obtinrent que 20 sièges seulement. Un résultat encore plus important fut l'émergence d'une alliance ultraconservatrice qui rejetait la plupart des idées aussi bien des panroumains que des moldovistes et demandait, entre autres choses, à ce que le russe soit la langue officielle de la République. Composée du Parti socialiste (l'héritier le plus direct de l'ancien Parti communiste de Moldovie) et du Mouvement Edinstvo-Unitatea (le successeur du Front uni de l'époque pérestroïkienne), l'Alliance reçut $22 \%$ des suffrages, ce qui en faisait le deuxième parti (avec 28 sièges) derrière les Agrariens Démocrates ${ }^{34}$. Aucun des neuf autres partis et alliances électorales ne pût franchir le seuil des $4 \%$ nécessaires pour entrer au Parlement ${ }^{35}$.

23 La raison du changement de discours récent du gouvernement sur la question identitaire est claire. En adoptant un nationalisme indigène moldove comme fondement de la république moldove, faisant ressurgir, non seulement la mémoire de l'éphémère république bessarabienne indépendante, mais aussi la notion d'une langue indépendante moldove, Snegur tentait de présenter son gouvernement, ainsi que luimême comme les garants de l'indépendance et de l'intégrité territoriale moldoves. 
C'est un thème qui réussit fort bien à la campagne. Deux-tiers des Moldoves / Roumains vivent dans des villages où ils représentent $80 \%$ de la population rurale totale ${ }^{36}$. Les enquêtes menées par William Crowther faisaient ressortir que moins de $10 \%$ de la population moldove / roumaine entendait soutenir l'unification avec la Roumanie dans le court ou long terme, et, lorsqu'ils avaient le choix entre les définitions ethniques "Roumain" ou "Moldove", $87 \%$ des locuteurs de la langue moldove / roumaine choisissaient la seconde ${ }^{37}$. Pareillement, une "enquête sociologique" soutenue par le gouvernement (La sfat cu poporul) menée une semaine après les élections, bien que criticable tant du point de vue scientifique que légal ${ }^{38}$, sembla donner des résultats clairement en faveur de l'indépendance, avec plus de $90 \%$ des participants votant "oui" pour une république indépendante dans ses frontières actuelles ${ }^{39}$.

Tout comme à la fin des années 1980, quand il pouvait jalonner une piste entre la radicalité des panroumanistes et l'intransigeance du premier-secrétaire du Parti communiste, Snegur tenta alors de faire son chemin entre les restes du Front et le puissant nouveau groupe Socialites-Edinstvo. Le premier poussant la Moldovie vers Bucarest et le second vers Moscou, un "moldovisme" indigène semblait le seul recours pour un gouvernement soucieux de maintenir sa propre indépendance. Pour Snegur en particulier, cultiver un nationalisme moldove indigène devrait lui assurer un résultat favorable lors des prochaines élections présidentielles, tandis que son principal concurrent sera l'actuel président du Parlement, Petru Lucinschi; tous les deux sont nés en Moldovie, mais Lucinschi a passé la plupart de sa carrière politique en dehors de la République et sera donc vulnérable aux attaques du titulaire de l'“autochtonie". La conception "moldoviste" de l'identité nationale place toutefois le président dans une impasse: non seulement elle lui aliène l'intelligentsia panroumaine, qui promet maintenant de maintenir le contentieux sur l'identité nationale en première place du discours politique, mais elle menace aussi d'ébranler les bases non-ethniques et civiques de l'État qui sont essentielles pour empêcher un conflit interethnique.

\section{La Moldovie face à ses minorités}

Ces quatre dernières années, Snegur et d'autres hauts fonctionnaires moldoves ont travaillé à souligner le caractère civique et multiethnique de l'État moldove afin d'apaiser les craintes des populations minoritaires pensant que la finalité de l'indépendance moldove est l'unification avec la Roumanie. Plus d'un tiers de la population moldove est constituée de minorités non moldoves / roumaines, et un nombre élevé de Slaves (Ukrainiens, Russes et Bulgares) et de Gagaouzes (Turcs orthodoxes) sont concentrés dans le sud et l'est de la Moldovie ${ }^{40}$. Depuis 1989, Chisinau a été impliquée dans une guerre froide, parfois chaude, avec deux républiques séparatistes proclamées en 1990, au sud (la République de Gagaouzie) et sur la rive orientale du Dniestr (la République transnistrienne moldove).

Les Gagaouzes et les Transnistriens craignirent d'abord que l'euphorie panroumaine qui secoua la république pendant la seconde moitié de l'année 1989 n'aboutisse à leur "roumanisation" forcée et à une unification rapide de la Moldovie avec la Roumanie. Les nouvelles lois sur la langue étaient leur principale source d'inquiétude. En 1989, moins de $4 \%$ des locuteurs moldoves / roumains non-autochtones considéraient le moldove / roumain comme leur seconde langue, tandis que près de $60 \%$ de la population moldove considérait le russe comme leur langue maternelle ou comme leur 
seconde langue ${ }^{41}$. Cependant, malgré les appels pour faire aussi bien du moldove / roumain, que du russe les langues officielles, on donna au russe le statut moins exaltant de «langue de communication parmi les nations» et de «langue de communication parmi les nations de l'Union Soviétique $\rrbracket^{42}$. L'utilisation de la même phrase (limba de comunicare intre natiuni/iazyk mezhnatsional'nogo obshcheniia) dans les deux contextes rendait la loi ambiguë : avec la destruction de l'Union Soviétique, il n'était pas clair que le russe ait encore une position officielle en Moldovie comme "langue de communication inter-ethnique » ou soit plutôt une langue à utiliser dans les relations avec les autres États successeurs. Plus important, les lois sur la langue définissaient une limite temporelle pour l'apprentissage du moldove / roumain, et elles prévoyaient un plan ambitieux pour une vérification mandatée de pratiquement tous les citoyens moldoves - des ministres du gouvernement aux magasiniers - en 1996.

En 1989 et 1990, le gouvernement du premier ministre Mircea Druc, dirigeant du Front Populaire, fit bien peu pour apaiser les peurs des séparatistes. Le gouvernement Druc fut désigné par le président Snegur en déférence au pouvoir du Front Populaire, et Druc utilisa sa position dans le gouvernement dominé par les Moldoves / Roumains pour appeler des volontaires à prendre les armes pour la défense de l'intégrité territoriale moldove. Une guerre totale avec les Transnistriens dans la première moitié de l'année 1992, aussi bien que des escarmouches périodiques avec les Gagaouzes, créèrent un climat de méfiance parmi les diverses parties en conflit et se traduisit par des pertes humaines et matérielles importantes. Depuis ce temps pourtant, la direction moldove travaille à apaiser les Gagaouzes et les Transnistriens en soulignant la nature nonethnique et citoyenne de l'État moldove. Les successeurs de Druc, spécialement le premier ministre actuel, Andrei Sangheli, ont dirigé leurs gouvernements d'une manière qui reflétait plus précisément le caractère multi-ethnique de la république. En fait, lorsque le gouvernement Sangheli fut formé au cours de l'été et de l'automne 1992, des portefeuilles furent spécialement réservé pour des ministres des régions séparatistes, bien que les Transnistriens déclinèrent l'offre. La perte de la grâce du Front après la démission de Druc en 1991 a été accélérée par le remplacement des ministres de la Défense et de la Sécurité de l'État en juillet 1992 pour leur traitement trop zélé de la crise transnistrienne. Le dernier panroumaniste déclaré en haute fonction tomba en janvier 1993 avec la démission forcée du président du Parlement moldove, Alexandru Mosanu, ainsi que de plusieurs de ses collègues du présidium.

La composition des hauts dirigeants politiques de Moldovie connut donc un immense changement : bien que les unionistes pan-roumains aient été capables de profiter de la vague de libération nationale pour assumer quelques-uns des plus hauts postes dans le gouvernement et au Parlement après 1989, leur inaptitude dans le traitement des désaccords inter-ethniques conduisit à leur remplacement par d'anciens communistes, ceux-là mêmes qu'ils avaient dénoncés comme "roumanophobes", et des communisants au sein du mouvement national. Au milieu de 1993, les postes de Président, Premier ministre, ministre des Affaires Étrangères et président du Parlement étaient tenus par d'anciens membres du Politburo. Plus spectaculairement, le remplaçant de Mosanu au poste de président du Parlement fut Petru Lucinschi, le pénultième premier-secrétaire du Parti communiste moldove, dont le retour politique lui valu le surnom de "Brazauskas moldove" 4 .

Le nouveau leadership moldove, en particulier le triumvirat Snegur-Sangheli-Lucinschi, chercha à établir un dialogue avec les séparatistes. Des rencontres informelles eurent 
régulièrement lieu avec Igor' Smirnov, Aleksandr Karaman et Grigore Marakutsa (respectivement : le Président transnistrien, le vice-président et le président du Soviet Suprême). La rhétorique de l'affrontement de Chisinau, dont des procès répétés à l'encontre de Smirnov, cessa, et Snegur accepta l'idée que la Transnistrie devait bénéficier d'un statut particulier au sein de la Moldovie. En fait, sur la base de l'accord signé par Snegur et Boris Eltsine en juillet 1992, établissant une force de maintien de la paix commune russo-moldovo-transnistrienne le long du Dniestr, le président tenta d'élaborer un arrangement amiable sur l'autonomie locale pour la région de la rive orientale. Une série de "principes de base" proposée par le gouvernement moldove reconnaissait les «caractéristiques historiques, sociales et culturelles » spécifiques de l'actuelle « République transnistrienne moldove ». Les cinq raions de la rive orientale et la ville de Tiraspol' étaient définis comme le «territoire transnistrien autoadministré » (Pridnestrovskaia samoupravliaemaia territoriia), avec un pouvoir de décision en ce qui concerne le budget, le salaire minimum, les taxes, les licenses d'importation et d'exportation, les relations économiques avec l'étranger, la santé publique et les services sociaux, la police, et dans d'autres domaines. Plus significativement, les lois sur la langue adoptées en 1989, principale source de mécontentement des minorités ethniques de Moldovie, ne seront pas appliquées en Transnistrie avant 2005. Cependant, la contre-proposition transnistrienne rejetait tout ce qui s'éloignait d'une confédération souple, avec peu de pouvoirs réservés aux autorités centrales ${ }^{44}$. Le Soviet Suprême transnistrien a déjà adopté ses propres lois sur la citoyenneté, établi des postes de douane le long du Dniestr et introduit sa propre monnaie (le suvorov) ${ }^{45}$.

De plus, la fédération russe soutint activement les Transnistriens dans leur lutte contre Chisinau et analysa le conflit comme le résultat du revanchisme de la Moldovie envers sa population russe. Plus d'un quart des 562000 Russes de Moldovie vivent en Transnistrie et les résultats des dernières élections russes semblent indiquer que leurs griefs recevront désormais une écoute encore plus sympathique de la part de Moscou.

En 1989, les griefs transnistriens étaient presqu'exclusivement liés aux lois sur la langue et la menace de l'unification avec la Roumanie. Comme les Unionistes furent progressivement purgés du gouvernement moldove et que la direction de Snegur assouplissait ses conditions, les demandes des Transnistriens se modifièrent sensiblement. Ce qui avait débuté comme un mouvement de prévention contre la roumanisation semblait désormais s'orienter vers un mouvement s'opposant à la démocratisation. Peu des changements sociaux et politiques effectifs à travers l'ancienne URSS touchèrent la Transnistrie, et la république maintint toutes les anciennes lois, les symboles et les vacances soviétiques, dont une interdiction d'écrire le moldove / roumain en alphabet latin. La région de la rive orientale était aussi devenue un refuge et un symbole pour ces groupes désillusionnés par la transition en Russie. Le ministre transnistrien de la Sécurité de l'État et les ministres de la Sécurité et de l'Intérieur sont tous d'anciens officiers OMON recherchés pour crimes en Lettonie et en Estonie, et des irréguliers transnistriens avaient été envoyés à Moscou pour participer à la défense de la Maison Blanche en octobre 1993. Les Moldoves espéraient que les événements d'octobre montreraient aux Russes l'empressement des Transnistriens à mordre la main qui les nourrit, mais Moscou s'abstint de faire un lien entre les soldats combattant à Moscou et le soutien ferme de la fédération russe à l'autodétermination transnistrienne ${ }^{46}$. La présence de la $14^{\text {ème }}$ armée russe, des troupes de l'ex-URSS à Tirapol', la capitale transnistrienne, complique encore plus la situation. Peu de progrès furent réalisés dans les relations moldovo-russes sur leur retrait, et le 
commandant de l'armée, le major-général Aleksandr Lebed' connu pour ne pas mâcher ses mots, prit une part active dans la vie politique transnistrienne. Il accusait souvent les Moldoves de chauvinisme ethnique et les Transnistriens de corruption, et il suggéra que le "précédent de Pinochet" pouvait fournir une solution à la crise moldovotransnistrienne ${ }^{47}$. Le pouvoir détenu par le commandant de la $14^{\text {ème }}$ armée s'illustrait par le fait que les Transnistriens faisaient souvent la queue devant son bureau pour lui soumettre des problèmes de hausse des loyers, de pensions impayées et d'autres problèmes domestiques; en fait, en 1992, Lebed' fut élu " homme de l'année ${ }^{48}$. Bien que la CSCE ait analysé le plan moldove pour l'attribution d'un statut spécial à la Transnistrie comme une incitation à la Russie de retirer la $14^{\text {ème }}$ armée ${ }^{49}$, Moscou est réticente à perdre les avantages stratégiques que lui procurent la présence de l'armée dans la région, notamment au vu de la dispute s'accentuant avec l'Ukraine ${ }^{50}$.

La direction de Snegur fit mieux dans les négociations avec les Gagaouzes. Une commission parlementaire sur les affaires gagaouzes fut formée en mars 1993 et un projet de loi sur une autonomie locale fut déposée en mai. Le projet désignait la région habitée par une forte proportion de Gagaouzes comme une circonscription administrative spéciale, Gagauz Eri (littéralement le "lieu gagaouze"), avec une large autonomie culturelle et administrative et le droit à l'autodétermination si la Moldovie devait " perdre sa souveraineté étatique » (c'est-à-dire en s'unifiant avec la Roumanie). En retour, les Gagaouzes devaient préserver l'intégrité territoriale de la République de Moldovie et respecter Chisinau comme l'autorité politique suprême de la République. Une contre-proposition avancée par le Soviet Suprême gagaouze, situé dans la ville de Comrat, insista sur la création d'une confédération moldove consistant en la Gagaouzie, la Transnistrie et une république moldove tronquée ${ }^{51}$. La Gagaouzie serait un État totalement souverain, gouverné par un président et un Parlement élus au suffrage direct, le Baskan et le Yusek Toplus, tandis que le gagaouze, le moldove / roumain, le russe et le bulgare seraient considérés comme des langues d'État égales ${ }^{52}$. Le projet de Chisinau ne parvint pas à obtenir le soutien du Parlement moldove d'avant 1994, mais la nouvelle Assemblée prévoyait dans son agenda de ré-examiner la proposition de loi.

Les relations entre Chisinau et Comrat commencèrent à s'améliorer significativement au début de 1994. Stepan Topal, le président de la Gagaouzie, applaudit le discours de Snegur au congrès "Chez Nous », le Soviet Suprême permit l'installation de bureaux de vote pour les élections parlementaires et à $L a$ sfat cu poporul de dépouiller les bulletins en Gagaouzie ; la direction gagaouze salua unanimement les résultats électoraux ${ }^{53}$. La section gagaouze de l'Académie des Sciences de Moldovie contribua au développement de l'alphabet latin pour le gagaouze écrit et à l'élaboration d'un programme pour améliorer le niveau de vie des 136000 Gagaouzes vivant dans le sud moldove ${ }^{54}$. Des réformes telles que l'ouverture d'une section gagaouze dans l'Institut pédagogique moldove, l'établissement d'une université gagaouze à Comrat et l'introduction de l'apprentissage de la langue natale dans les villages gagaouzes ont servi à apaiser les membres les plus radicaux du Soviet Suprême gagaouze. Des aides en provenance de la Turquie et de la Bulgarie sont aussi arrivées aux Gagaouzes et aux Bulgares du sud de la Moldovie, en particulier à l'université de Comrat, dans le but de convaincre les minorités que, même dans une Moldovie non-fédérale, leurs droits culturels seraient respectés ${ }^{55}$. Bien que l'amélioration de la situation soit en partie due au ton plus conciliant de Chisinau, le gouvernement gagaouze a lui-même eu un grand rôle en faisant de nécessité vertu. Les Gagaouzes n'ont pas eu accès aux caches d'armes dont disposent les Transnistriens (via la $14^{\text {ème }}$ armée), ils vivent dans la région moldove la 
plus pauvre et ne menacent donc pas l'État au niveau de la plupart de ses ressources industrielles et énergétiques (comme le font les séparatistes de Transnistrie). Ils comptent toujours sur Chisinau pour subventionner le budget local, et ils se montrés incapables d'établir un contrôle effectif sur les cinq raions désignés comme république gagaouze. De fait, les Gagaouzes ne forment une majorité que dans deux des raions, et la population moldove et bulgare locale a bien peu soutenu la direction gagaouze. De la même façon, l'absence générale d'ordre prévalant dans le "sud sauvage" de la Moldovie s'est révélée au-dessus des capacités de quiconque, Moldove ou Gagaouze, à contrôler.

Snegur s'est donc révélé relativement victorieux dans ses tentatives d'apaiser les craintes causées par le zêle panroumain du Front en 1989-1990. Un élément important de sa stratégie a été de souligner le caractère multiethnique de l'État moldove. La loi sur la citoyenneté adoptée en 1991 est parmi les plus libérales de l'ancienne Union soviétique et quelques-unes des premières actions du nouveau Parlement postsoviétique ont été de suspendre le contrôle de la connaissance de la langue moldove / roumaine prévu par les lois sur la langue de 1989 et d'affirmer l'adhésion de la Moldovie à la CEI. Toutefois, la conception civique de la moldovité présentée aux séparatistes diffère de la conception ethnique que Snegur met en avant dans ses disputes avec les panroumains. Promouvoir différentes conceptions selon l'auditoire a certainement mis en lumière la capacité du gouvernement à adapter le message politique au milieu politique. Mais les confrontations internes avec les panroumains ont obligé Snegur à s'étendre sur un sujet qu'il aurait dû laisser de côté, à savoir, l'ethnicité des Moldoves et leur situation d'habitants « les plus légitimes » de la région $\mathrm{du}$ Prout-Dniestr. Les relations avec la Roumanie, qui voit dans les intellectuels panroumains à Chisinau ses alliés naturels, ont compliqué la situation en poussant le gouvernement de Snegur toujours plus près de la défense d'une ethnicité moldove distincte comme fondement de l'État moldove.

\section{Les relations Roumanie - Moldovie}

L'irrédentisme est un élément nécessaire dans le programme d'un parti dans le paysage politique en Roumanie. Des groupes aussi radicalement différents que le "bloc nationaliste" au Parlement, le Mouvement pour la Roumanie, inspiré de la Garde de Fer, et la coalition Convention Démocratique, ont appelé à la réintégration des anciens territoires roumains formant actuellement une partie de l'Ukraine et de la Moldovie ; des commentateurs en Roumanie ont même invoqué ce qu'on pourrait appeler le "complexe du Kosovo", c'est-à-dire l'idée qu'une partie du territoire historique est mystiquement liée au bien-être collectif de l'ethnos ${ }^{56}$. Pour beaucoup de Roumains, le retour de la "terre sacrée" (sfintul pamint) de Bessarabie - du fait de ses rapports avec Stefan le Grand et le poète national, Mihai Eminescu - est essentiel pour effacer les conséquences de la deuxième guerre mondiale et l'héritage du communisme.

Le président roumain Ion Iliescu et le Parti de la Social-Démocratie de Roumanie (PSDR, l'ancien Front de Salut National Démocratique) ont été extrêmement prudents dans leurs relations avec les Moldoves. Toutefois, depuis les élections parlementaires de septembre 1992, le PSDR ne disposait plus de suffisamment de sièges au Parlement pour pouvoir faire passer ses programmes sans l'aide du puissant bloc nationaliste, composé de trois partis officiellement attachés à l'unification roumano-moldove. Les nationalistes - le Parti de la Grande Roumanie, le Parti de l'Unité Nationale Roumaine et 
le Parti Travailliste Socialiste - sont en général plus préoccupés par les "conspirations" hongroise et juive que par la résurrection d'une grande Roumanie, mais le besoin pour Iliescu de les récompenser périodiquement pour leur soutien lui laisse peu de marge de manoeuvre dans les relations avec la Moldovie. En fait, la nommination d'extrémistes dans diverses places au ministère de la culture au milieu de l'année 1993 et la perpétuation de la controverse entourant la formation d'un gouvernement de coalition ont révélé l'étendue de la dette du PSDR envers de tels groupes ${ }^{57}$.

Après l'échec de la ratification de l'adhésion à la CEI, la Roumanie fut prompte à féliciter la Moldovie pour son choix. Iliescu analysa l'échec de la motion comme un vote "pour le développement et le renforcement de l'indépendance (...) dans le cadre naturel de l'espace européen, avec lequel [la Moldovie] est liée non seulement par les relations historiques avec la Roumanie, mais aussi par les aspirations actuelles à l'émancipation $»^{58}$. Il offrit aussi aux Moldoves une aide substantielle pour payer l'arriéré des dettes de pétrole à la fédération russe. Une série de liens officiels existent entre les gouvernements de Chisinau et Bucarest, dont des groupes de travail de haut niveau sur les relations bilatérales, la Roumanie étant le plus grand partenaire économique en dehors de la CEI. Cependant, les Moldoves ne manquent aucune occasion de souligner l'existence de deux États distincts. Comme Petru Lucinschi le déclara durant sa visite en Roumanie en juin 1993, le fait que la Roumanie et la Moldovie partagent « une histoire commune centenaire, une seule langue [et] une culture commune » ne doit pas conduire à des questions d'unification "irréalistes et abstraites ». Lucinschi utilisa aussi l'ethnonyme "Moldove" pour décrire la nationalité titulaire de la république, une formulation qui fit enrager une grande partie de son auditoire en Roumanie ${ }^{59}$. De la même manière, dans une allocution du ministre moldove des affaires étrangères de l'époque, Nicolae Tiu, aux Nations unies, les références à la Roumanie étaient manifestement absentes et la plus grande partie de son discours constituait une critique du soutien de la fédération russe aux Transnistriens ${ }^{60}$. Une telle omission avait une signification particulière dans le cadre de l'Assemblée générale de l'ONU : afin d'éviter l'émergence de prétentions territoriales sur la région "moldove" de la part de la Roumanie (la moitié occidentale de l'ancienne Principauté de Moldovie), le délégué de Chisinau représente officiellement la "République de Moldovie" et, d'après la disposition alphabétique des sièges, il est assis près du représentant de la Roumanie ${ }^{61}$.

Plus spectaculairement, le discours de Snegur au congrès Chez nous en février 1994 et l'enquête La sfat cu poporul qui lui fit suite donnèrent lieu à toute une série d'attaques au vitriol en provenance de Bucarest. Le président de la Chambre des Députés roumaine publia une déclaration officielle dénonçant la "politique anti-roumaine " menée par Snegur ${ }^{62}$, tandis que le Parti Démocratique de Roumanie (l'ancien Front de Salut National) condamnait les discours de Snegur et de Motpan comme "manquant de culture, formulés pour des raisons purement électorales, contraires aux vérités historiques et, par conséquent, contraires aux intérêts des Roumains [vivant] entre le Prout et le Dniestr ${ }^{63}$. Des déclarations similaires suivirent de la part d'autres partis roumains et de membres du gouvernement - dont la proclamation par le ministre des affaires étrangères que « seulement l'histoire » déciderait du destin de la Moldovie -, et des personnalités politiques moldoves telles que Motpan et le premier ministre Sangheli reprochèrent aux Roumains d'insulter le peuple moldove et d'attaquer la légitimité étatique de la Moldovie ${ }^{64}$. 
39 L'attitude condescendante de la Roumanie a, depuis le début de 1994, poussé la direction moldove dans l'adoption d'une ligne plus ferme sur l'identité moldove. En fait, une des raisons de la franche condamnation par Snegur des panroumains lors de la campagne pour les élections de février était certainement une tentative d'affirmer sa position vis-à-vis de Bucarest. En fait, son discours Chez nous contenait un appel direct à ses auditeurs roumains : "Notre peuple moldove ne veut plus être une marchandise d'échange ou la victime de quelqu'un d'autre. Ils ne veulent plus entendre [quelqu'un d'autre] affirmer que leur pays fait partie de son territoire (...), comme s'il ne disposait pas de ses authentiques propriétaires ${ }^{65}$. Tenir tête aux Roumains et aux panroumains de Chisinau est essentiel à l'avenir politique de Snegur et a sans aucun doute aidé au résultat électoral des Agrariens. Le plus souvent, les politiciens tant moldoves que roumains se contentent de répondre à la vox populi : ainsi que les dernières élections et les "enquêtes sociologiques" l'ont souligné, les politiciens moldoves sont, pour partie, jugés sur leur capacité à répondre à une forte ligne moldoviste et indépendantiste, tandis que les politiciens de Bucarest doivent, d'une manière similaire, plaider pour la réintégration des territoires perdus en 1940.

40 Cependant, chaque obligation a son prix. Pour les Roumains, la rhétorique sur l'irrédentisme ternit leur image à l'étranger et mine leur volonté de représenter une source de stabilité en Europe du sud-est. Pour les Moldoves, les conséquences de leur insistance sur le problème de l'identité sont potentiellement plus graves. D'une part, les intellectuels panroumains de Chisinau et les grondements irrédentistes en provenance de Bucarest ont obligé Snegur et les Agrariens à présenter une identité moldove distincte - basée sur des caractéristiques linguistiques indigènes et l'éphémère République bessarabienne indépendante - en tant que fondement de l'État moldove actuel. D'autre part, la menace de séparatisme territorial des Gagaouzes et des Transnistriens a contraint Snegur et ses associés à insister sur le concept d'une citoyenneté moldove - basée sur l'appréciation de la complexité ethnique de la région comme le fondement étatique de la Moldovie. Tandis que cette tension entre les conceptions ethnique et civique de l'identité nationale n'est certainement pas la première en Moldovie, l'histoire du pays et l'actuel aménagement politique ont rendu le jeu de l'indécision entre ces deux conceptions particulièrement difficile.

41 Les élections de février 1994 et l'“enquête sociologique" de mars ont mis en évidence que peu de Moldoves se perçoivent comme des Roumains et qu'encore moins ont quelque velléité que ce soit pour la recréation d'une "Grande Roumanie". En fait, le seul parti à faire uniquement campagne sur l'unification immédiate, le Parti National Chrétien, arriva dernier à l'élection, avec moins de 6000 voix. Les intellectuels de Moldovie, ainsi que de nombreux observateurs occidentaux, n'ont offert qu'une explication simple de ce phénomène: ils perçoivent l'utilisation continuelle de l'ethnonyme "Moldove" par une large partie de la population comme une preuve de l'ampleur de la "dénationalisation" menée par les Soviétiques et perpétuée par leurs successeurs. D'après le critique littéraire respecté, Ion Ciocanu, les intérêts politiques cachés des Agrariens sont à condamner parce qu'ils masquent la « vérité scientifique et historique » que les Moldoves sont en fait des Roumains, parlent le roumain, et forment la « population autochtone » légitime (populatia bastinasa) de Moldovie ${ }^{66}$. Si ce n'était les machinations des Agrariens, disent-ils, les Moldoves se réveilleraient avec la roumanité en eux. 


\section{Conclusion}

42 Les raisons de l'échec du panroumanisme sont certainement beaucoup plus complexes. En fait, la vrai surprise à propos de l'identité moldove n'est pas le fait que les Moldoves aient rejeté leur prétendue roumanité, mais bien plutôt que de nombreux observateurs occidentaux, aussi bien journalistes qu'universitaires, aient prédit qu'ils l'embrasseraient. L'existence de la Bessarabie au sein de l'Empire russe durant les années de la formation de la conscience nationale roumaine, l'héritage déshonorant de la direction roumaine entre les deux guerres, la politique officielle soviétique après 1924 et 1940, la situation économique peu enviable de la Roumanie, des liens commerciaux et culturels continus avec les anciennes Républiques soviétiques, et l'attitude condescendante avec laquelle les Moldoves continuent d'être traités par Bucarest sont autant de facteurs qui ont renforcé une identité moldove indépendante du panroumanisme des intellectuels de Chisinau.

43 Comme j'ai tenté de le montrer, s'opposer aux arguments des panroumains a fourni à l'actuel gouvernement un considérable capital politique. Vis-à-vis de la population moldove / roumaine, le gouvernement Snegur a pu se présenter comme le défenseur de la souveraineté nationale et d'une identité moldove distincte, une image qui a séduit la paysannerie moldove. Avec les Gagaouzes et les Transnistriens, le gouvernement a forgé un concept non-ethnique de la citoyenneté comme rempart aux aspirations panroumaines de l'intelligentsia moldove / roumaine, une tactique qui a aidé à réduire les tensions entre le centre et les régions séparatistes.

44 L'utilisation par le gouvernement de la question identitaire dans les deux milieux a eu le dangereux effet de mettre en lumière la tension existant entre les conceptions ethnique et civique de la moldovité et a instauré les débats sur l'identité nationale et l'État moldove comme composantes clefs du processus politique. Il y a plusieurs raisons de croire qu'ils le demeureront. Premièrement, si le bloc Socialistes-Edinstvo dans le nouveau Parlement poursuit son programme politique, il remettra de plus en plus en question l'existence de la Moldovie aux frontières de la CEI, poussera le gouvernement à intégrer la zone du rouble, permettra à Moscou une présence militaire permanente en Transnistrie et remettra en cause les réformes initiées depuis 1989. Snegur, Sangheli et Lucinschi, malgré leur passé politique, s'y sont, bien entendu, fermement opposés. Le gouvernement pourrait alors trouver dans un nationalisme indigène moldove, du style de celui préconisé par le congrès Chez nous, un moyen de mobilisation contre l'alliance ultraconservatrice.

Deuxièmement, puisque le nouveau Parlement examine le projet de loi sur l'autonomie locale pour la Gagaouzie et la Transnistrie et met un point final à une nouvelle proposition de constitution, les fondements de l'État moldove vont sans aucun doute se préciser. Si la Moldovie doit être définie comme un État "national" (et comment cette "nation" devrait être dénommée), le degré de l'autonomie à garantir aux régions séparatistes et les symboles nationaux à inscrire dans la constitution sont autant de questions invitant au débat sur les notions fondamentales de la moldovité. De plus, les panroumains, à Chisinau aussi bien qu'à Bucarest, ont refusé que les Gagaouzes puissent être vus autrement que comme des touristes dans l'“espace roumain" traditionnel; selon eux, accorder aux Gagaouzes le droit constitutionnel de faire sécession en cas d'unification moldovo-roumaine revient purement et simplement à placer une bombe nationale au centre de la grande Roumanie historique ${ }^{67}$. 

par les élections de février, mais les panroumains et leurs alliés n'ont pas disparu en tant que force politique active. En premièr lieu, le vieux Front Populaire a donné naissance à d'autres partis politiques, qui, bien que d'avis divergents quant à l'unification moldovo-roumaine, n'en sont pas moins unis dans leur opposition au gouvernement mené par les Agrariens, et voient dans la question identitaire une arme formidable contre celui-ci ${ }^{68}$. Le Parti de la Réforme et le parti Travailliste Démocratique, bien qu'encore jeunes, en sont de bons exemples. Les deux disposent d'une base électorale confortable, parmi les Moldoves / Roumains instruits et urbains, nombre d'entre eux étant impliqué dans le secteur économique privé en expansion. Pour ces groupes, Snegur et l'ancienne élite du parti utilisent essentiellement la notion d'une identité moldove distincte afin de s'allier la paysannerie, d'affermir leur position au pouvoir dans la république et de bloquer les réformes économiques qui conduiraient à l'accroissement des élites urbaines. Comme antidote, les entrepreneurs et les managers moldoves / roumains des entreprises nouvellement privatisées ont souligné la "roumanité" de la république moldove, se rapprochant des panroumains, déclarant les Agrariens propriétaires démodés de la période communiste et accusant le gouvernement Snegur de perpétuer le mythe de deux peuples romains orientaux distincts. Donc, alors que le Front et le Congrès de l'Intelligentsia sont opposés à la position du gouvernement contraire à la "vérité scientifique et historique», les entrepreneurs moldoves / roumains le perçoivent comme un obstacle à l'éviction des apparatchiki de l'époque soviétique. De toute évidence, le Parti de la Réforme et le Parti Travailliste Démocratique restent assez calmes à propos de l'unification avec la Roumanie, mais l'affirmation de la roumanité de la république moldove demeurera certainement un thème majeur de leurs stratégies politiques.

Plus important, des panroumains dévoués contrôlent actuellement des institutions clefs, telle que l'Université de Chisinau, divers instituts de l'Académie des Sciences et l'Union des Écrivains. La purge de l'élite culturelle de la période soviétique après 1989 a amené au pouvoir des académiciens et des bureaucrates culturels moldoves / roumains plus jeunes voués aux idées $d u$ panroumanisme du Front et du Congrès de l'Intelligentsia. Ils se rendent fréquemment à Bucarest et Iasi, ont des contacts étroits avec les intellectuels roumains et, comme leurs ancêtres unionistes de 1918, considèrent comme leur devoir de réveiller l'esprit roumain dans la paysannerie somnolente. Si l'on se tourne vers le futur, cette scission entre 1'“intelligentsia créative" et le reste de la société moldove est le plus grand garant de la perpétuation de la saillance de la politique identitaire. Tant que les écoliers et les étudiants moldoves continueront d'étudier la littérature roumaine, de chercher les traits communs entre les deux rives du Prout et d'apprendre la perfidie de la "dénationalisation" soviétique après 1940, la question de l'identité nationale continuera d'être un des moteurs de la politique moldove. 


\section{NOTES}

1. Bien que la république soit mieux connue en occident sous le nom de Moldavie (une anglicisation du nom russe - "Moldavia"), elle est connue sous le nom de Moldova ou Moldova Sovietica par les locuteurs roumains. Dans cet article, j'utilise le terme, plutôt peu maniable, de "moldove / roumain" en faisant référence à la langue officielle et la nationalité nominale de Moldovie ["moldove" seul ayant alors trait à la nationalité et n'ayant aucune référence nationale]. Les langues littéraires de Chisinau et de Bucarest sont aisément intercompréhensibles, mais, ainsi que je le soutiens plus bas, la façon dont les Moldoves s'appellent et appellent leur langue est une question politique, et non pas linguistique.

2. Dorosh (Lina), « Vviazalsia v skhvatku », Grazhdanskii mir, 08/08/93.

3. «Deklaratsiia gruppy narodnykh deputatov Respubliki Moldova», Nezavisimaia Moldova, $14 / 08 / 93$.

4. Bien qu'il soit toujours problématique de définir des concepts tels que "nation" ou "identité", j'entends, ici, le terme "identité nationale" comme désignant l'appareil complexe de noms, langues, légendes, histoires et autres caractéristiques communes propres acceptées comme fondamentales dans l'auto-définition collective d'une population humaine donnée au sein d'un territoire délimité, et "politique identitaire" comme signifiant les luttes entre les élites culturelles et politiques à propos de l'authenticité de versions rivales sur l'identité nationale (cf. Bourdieu (Pierre), Language and Symbolic Power, Cambridge : Harvard University Press, 1991).

5. Cantemir (Dimitrie), Descrierea Moldovei, Chisinau : Hyperion, 1992, pp. 12-13.

6. Le nom "Bessarabie" était, en fait, mal approprié. La dynastie Basarab, de laquelle il provient, avait dirigé des régions de Valachie, et non de Moldovie, aux XIV ème et XV ${ }^{\text {ème }}$ siècles.

7. Plusieurs études classiques en langue roumaine de la période s'étendant jusqu'en 1918 ont été récemmement rééditées. Cf. Boldur (Alexandru), Istoria Basarabiei, Bucuresti : Victor Frunza, 1992 ; Nistor (Ion), Istoria Basarabiei, Bucuresti: Humanitas, 1991; Ciobanu (Stefan), Cultura romaneasca in Basarabia sub stapinirea rusa, Chisinau: Editura Enciclopedia "Gheorghe Asachi", 1992 ; idem, Unirea Basarabiei, Chisinau : Universitas, 1993.

8. Cf. Stoian (Stelian), "Viata politica din Basarabia in perioada de autonomie provizorie ", Revista de istorie a Moldovei, (2), 1992.

9. Manuila (Sabin), Studiu etnografic asupra populatiei Romaniei, Bucuresti : Editura Institutului Central de Statistica, 1940, pp. 54, 56.

10. Martonne (Em. de), What I Have Seen in Bessarabia, Paris : Imprimerie des Arts et des Sports, 1919, p. 10 ; Clark (Charles Upson), Bessarabia: Russia and Roumania on the Black Sea, New York: Dodd, Mead and Company, 1927, p. 82 ; Baerlein (Henry), Bessarabia and Beyond, London: Methuen and Co., Ltd., 1935, pp. 171-172.

11. Clark (Charles Upson), op.cit., p. 287-290. Les principautés roumaines adoptèrent l'alphabet latin au milieu du XIX ${ }^{\text {ème }}$ siècle, mais l'alphabet cyrillique fut conservé (et même préféré au latin) par les Moldoves dans la partie russe de la Bessarabie (cf. Livezeanu (Irina), «Moldavia, 1917-1990 : Nationalism and Internationalism Then and Now ", Armenian Review, 43 (2-3), 1990).

12. Vsesoiuznyi perepys liudnosti 1926 roku, Moskva : Vydannia TsSU Soiuzu RSR, 1929, (11), p. 30.

13. Sur les modifications démographiques et la politique culturelle durant cette période, cf. Livezeanu (Irina), «Urbanization in a Low Key and Linguistic Change in Soviet Moldovia, Part. I ", Soviet Studies, 33 (3), 1981 ; idem, « Urbanization in a Low Key and Linguistic Change in Soviet Moldovia, Part. II », Soviet Studies, 33 (4), 1981 ; King (Charles E.), « Soviet Policy in the Annexed East European Borderlands: The Case of Moldova» in Holtsmark (Sven), Westad (Odd Arne), Neumann (Iver), eds., The Soviet Union in Eastern Europe, 1945-1989, London: Macmillan, 1994 ; 
Meurs (Wim van), The Bessarabian Question in Communist Historiography, Boulder : East European Monographs, 1994.

14. Actele legislative ale R.S.S. Moldovenesti cu privire la decretarea limbii moldovenesti limba de statsi revenirea ei la grafia latina, Chisinau : Cartea Moldoveneasca, 1990, pp. 3, 5.

15. «Declaratia de independenta a Republicii Moldova », Romania Libera, 28/08/91.

16. Sur le mouvement national, cf. Eyal (Jonathan), « Moldovians », in Smith (Graham), ed., The Nationalities Question in the Soviet Union, New York: Longman, 1990 ; Crowther (William), «The Politics of Ethno-National Mobilization : Nationalism and Reform in Soviet Moldovia », Russian Review, 50, april 1991.

17. « Documentul final al Marii Adunarii Nationale », Literatura si Arta, 31/08/89.

18. Iurie Rosca, président du Comité Exécutif du Front Populaire Chrétien Démocrate de Moldovie, entretien avec l'auteur (Chisinau, 15/04/93).

19. Programul Frontului Popular Crestin Democrat, Chisinau, 1992, p. 8.

20. Socor (Vladimir), "Why Moldova Does Not Seek Reunification with Romania », RFE/RL Research Report, 31/01/92, p. 30. Alors que le Front pouvait attirer des centaines de milliers de Moldoves à sa "Grande Assemblée Nationale" en août 1989, une centaine seulement de supporters participèrent à des meetings similaires en été 1993.

21. Le président du Front, Mircea Druc, participa même aux élections présidentielles roumaines de septembre 1992, sur l'unique plateforme de la réunification moldovo-roumaine. Il reçut seulement $2,75 \%$ des suffrages, réalisant le plus mauvais score électoral des six candidats.

22. "Statutul Congresului Intelectualitatii », Literatura si Arta, 08/04/93.

23. Gamova (Svetlana), « Parlament Moldovy na grani samorospuska », Izvestiia, 29/01/93.

24. $32 \%$ des députés agrariens dans le Parlement actuel dirigent des fermes collectives ou des entreprises agro-industrielles. $28 \%$ sont d'anciens ministres (dont le Premier ministre Andrei Sangheli), des membres de haut-rang de l'ancien Parlement ou des dirigeants de gouvernements locaux. Le président du Parlement (Petru Lucinschi) et les deux vice-présidents (Dumitru Motpan et Nicolae Andronic) sont aussi des Agrariens Démocrates.

25. Tout l'approvisionnement moldove en gaz et en pétrole brut, $98 \%$ de l'approvisionnement en pétrole, $68 \%$ de celui en diesel et $36 \%$ de celui en charbon proviennent de la seule Russie. En 1992, $75 \%$ des exportations moldoves se sont réalisées en direction des anciennes républiques soviétiques, qui ont fourni à la Moldovie 78 \% de ses importations ("Ekonomicheskii soiuz: "pro" i "contra" ", Nezavisimaia Moldova, 07/08/93 ; Rotar (V.), "Parlament sdelal vse, chto mog?", Nezavisimaia Moldova, 24/08/93).

26. "Sovmestnaia deklaratsiia », Nezavisimaia Moldova, 05/08/93.

27. «Parlament razoshelsia s SNG », Nezavisimaia Moldova, 07/08/93.

28. Cf. les programmes des partis politiques dans Moldova Suverana, 05/02/94 et Respublika (4), 1994, pp. 4-5.

29. L'organisation n'a aucun rapport avec le groupe roumain de même nom qui l'a même accusée d'imposture («Spor ni o chem », Nezavisimaia Moldova, 30/12/93).

30. «Santem $[s i c]$ poate la cea mai hotaratoare rascruce din istoria neamului nostru », Pamint si Oameni, 12/02/94 ; Shatokhina (Elena), «Drutse i "Gazprom" - za Snegura », Moskovskie Novosti, 13-20/02/94.

31. Snegur (Mircea), «Republica Moldova este tara tuturor cetatenilor sai », Pamint si Oameni, $12 / 02 / 94$.

32. "O inventie a regimului comunist», Moldova Suverana, 12/02/94; "Sa respectam adevarul stiintific si istoric », Plus-Minus, février 1994, p. 8.

33. «Pericolul aservirii politice a vesnicelor adevaruri », Plus-Minus, février 1994, p. 8.

34. "Okonchatel'nye itogi Parlamentskikh vyborov », Moldova Suverana Daidzhest, 18/03/94.

35. La loi électorale post-soviétique stipulait que toute la République moldove formait une seule circonscription électorale, dont les députés étaient élus selon le système de liste close. Ainsi, 
puisque les candidats n'étaient liés à aucune circonscription régionale définie, le gouvernement soutenait que les régions séparatistes étaient représentées dans le nouveau Parlement, même si peu d'électeurs de ces régions participaient réellement au vote.

36. Republica Moldova, Chisinau : Universitas, 1992, p. 16.

37. Crowther (William), "The Politics of Ethnic Confrontation in Moldova», (communication présentée à la conférence du Woodrow Wilson Center, High Conflict / Low Conflict : Six Case Studies, Washington D.C., 28-30/06/93).

38. La sfat cu poporul ne fut ni un référendum, ni une enquête de l'opinion publique. La formulation de la question rendait difficile une réponse totalement affirmative ou négative, et, $\mathrm{du}$ fait qu'elle contenait plusieurs sous-questions dans une seule phrase, il était impossible de déterminer à quelle portion de la question générale les sondés répondaient (« Apropo », Dnevnoi ekspress, 22/02/94).

39. "Poporul si-a spus cuvîntul ", Moldova Suverana, 12/03/94.

40. D'après le recensement de 1989 , la population totale de la Moldovie se composait de $64,5 \%$ de Moldoves / Roumains, 13,8 \% d'Ukrainiens, 13,0\% de Russes, 3,5\% de Gagouzes et 2,0\% de Bulgares, le reliquat comprenant des juifs, des Tsiganes et d'autres nationalités. Dans les cinq raions à l'est du Dniestr (Transnistrie), les Moldoves / Roumains forment la majorité de la population (40\%), le reste se composant principalement d'Ukrainiens, de Russes et de Bulgares. Dans les cinq raions les plus au sud (Gagaouzie), les Gagaouzes forment le groupe le plus nombreux (47 \% de la population), suivi des Moldoves / Roumains et des Bulgares.

41. Economia Nationala a Republicii Moldova, 1991, Chisinau: Departamentul de Stat pentru Statistica, 1992, pp. 78-79.

42. Actele legislative, 1990 , p. 6.

43. Portnikov (Vitalii), " Moldavskii Gorbatchev' okazyvaetsia " moldavskim Brazauskasom " ", Nezavisimaia gazeta, 06/02/93.

44. Cf. l'addendum au «Report of the CSCE Human Dimension Mission to the Republic of Moldova (January 30-February 4, 1993) », CSCE, 1993, pp. 31-37.

45. Le "suvorov" est le rouble russe de 1961-1992, avec un poinçon spécial à l'effigie du fameux maréchal. Les nouveaux roubles russes sont aussi en circulation, mais le leu moldove (introduit en novembre 1993) n'est pas considéré comme monnaie légale («"Valuta" tiraspoleana ", Moldova Suverana, 20/01/94 ; « Nemilost' k moldavskomu leiu », Nezavisimaia Moldova, 24/11/93).

46. RFE/RL, Daily Report, 08/10/93.

47. Tsyba (Tat'iana), «Esli Vaniu shlepnut, eto nevosstanovimo », Komsomol'skaia pravda, 27/04/93. Lebed' est un fervent supporter de la présence de la $14{ }^{\text {ème }}$ armée en Transnistrie, mais il n'a montré aucune affinité avec la direction transnistrienne. Cf. King (Charles), « Moldova and the New Bessarabian Questions », World Today, july 1993.

48. Branimir Radev, chargé d'affaires bulgare en Moldovie, entretien avec l'auteur (Chisinau, 26/04/93) ; RFE/RL, Daily Report, 09/09/93.

49. «Doklad n¹3 missii SBSE v Moldove ", Makler-Telegraf, 03/02/94.

50. Pour un traitement détaillé de la crise transnistrienne et le rôle de la $14^{\text {ème }}$ armée, cf. Orr (Michael), « $14^{\text {th }}$ Army and the Crisis in Moldova », Jane's Intelligence Review, june 1992 ; Kolstø (Pål), Edemsky (Andrei), Kalashnikova (Natalya), «The Dniester Conflict: Between Irredentism and Separatism », Europe-Asia Studies, 45 (6), 1993.

51. Burlaca (Alexandru), «Gagauzia si Transnistria au propus crearea unei "Confederatii moldovenesti" ", Republica, 06/04/93.

52. Tago (Aleksandr), "Komrat - za federativnoe ustroistvo Moldovy ", Nezavisimaia gazeta, 17/02/93; "Komrat: proekt zakona o funktsionirovanii iazykov», Nezavisimaia Moldova, $05 / 08 / 93$.

53. "O biudzhete, vyborakh i drugom ", Komratskie vesti, 19/02/94 ; Golia (G.), "Veriu v razum novykh politikov », Kishinevskie novosti, 19/03/94. Les Gagaouzes menèrent leur propre enquête 
sociologique, parallèlement à celle de La sfat cu poporul. Plus de $85 \%$ des participants soutenaient un "territoire national" gagaouze autonome au sein d'une Moldovie indépendante (Butnaru (Val), "Agrarienii vor avea de platit niste polite preelectorale », Observatorul de Chisinau, 22/03/94).

54. Gavril Gaidargi, directeur adjoint de l'Institut moldove des minorités nationales, entretien avec l'auteur (Chisinau, 08/04/93).

55. La Turquie a montré un intérêt particulier dans le problème gagaouze, perçu comme partie intégrante de ses efforts pour établir des liens avec les populations turques de l'ancienne URSS. Deux livres sur les Gagaouzes, ce qui est assez rare en dehors de l'URSS, ont été récemment publiés à Ankara : Güngör (Harun), Argunsah (Mustafa), Gagauz Türkleri : tarih, dil, folklor ve halk edebiyatl, Ankara : Kültür Bakanllgl, 1991 ; idem, Gagauzlar: dünden bugüne, Ankara : Elektronik Iletisim Ajansi, 1993.

56. La presse roumaine a souvent publié de tels discours durant la guerre transnistrienne de 1992. Cf. par exemple Artenie (Tudor), «Sint mai buni si mai chinuiti ca noi », Romania Libera, 04-05/04/92 ; Barsan (Victor), Masacrul inocentilor : razboiul din Moldova, 1 martie - 29 iulie 1992, Bucuresti : Editura Fundatiei Culturale Romane, 1993.

57. Cf. Shafir (Michael), "Romanian Prime Minister Announces Cabinet Changes », RFE/RL Research Report, 24/09/93; Ionescu (Dan), Shafir (Michael), «Romanian Government Reorganized », RFE/RL Research Report, 01/04/94.

58. «Deklaratsiia prezidenta Rumynii », Nezavisimaia Moldova, 14/08/93.

59. «Vystuplenie Predsedatelia Parlamenta Respubliki Moldova», Nezavisimaia Moldova, 14/07/93.

60. "Vystuplenie ministra inostrannykh del Respubliki Moldova», Nezavisimaia Moldova, 13/10/93. Tiu a, depuis, été nommé ambassadeur à Washington.

61. Je remercie Jonathan Eyal d'avoir porté ce fait à ma connaissance.

62. «Declaratii de o gravitate extrema », Moldova Suverana, 16/02/94.

63. "Contrar adevarului istoric », Moldova Suverana, 16/02/94.

64. Associated Press (Bucarest), 07/03/94 ; RFE/RL, Daily Report, 4,8 et 17/03/94.

65. Snegur (Mircea), art.cit., p. 3.

66. Ciocanu (Ion), « Cine nu stie ceea ce stie toata lumea?", Literatura si Arta, 15/04/93.

67. Ce point de vue est vigoureusement défendu dans Iuncu (R.), ed., $K$ voprosu o gagauzskoi avtonomii, Chisinau : Cartea Moldoveneasca, 1990.

68. Pour une vision plus précise de ces groupes, cf. Opinca (Valeriu), Gorea (Igor), «Stanovlenie mnogopartiinosti v Respublike Moldova », Grazhdanskii mir, 23/04/93.

\section{RÉSUMÉS}

L'indépendance de la Moldovie est considérée par les groupes panroumains comme le premier pas vers la réunification à la Roumanie. Les groupes "moldovistes ", quant à eux, bien que ne remettant pas en cause la langue et la culture communes, estiment que l'histoire condamne la Moldovie à former un Etat. S'ajoute, à ce débat sur l'identité de la nation, le problème de régions sécessionnistes dans le sud et l'est. La langue, l'unification ou non à la Roumanie, les relations avec la Russie, la politique à adopter vis-à-vis des minorités sont autant de débats qui ont structuré le champ politique dans les premières années de l'après-communisme en Moldovie. 
Toutefois, l'attitude condescendante de la Roumanie a poussé les dirigeants vers une affirmation de l'identité moldove, ce qui représente un capital politique considérable.

For "pan-Romanianists", the independence of Moldova was the first step toward the reunification with Romania. On the contrary, « Moldovanists », though they do not deny a shared language and culture, maintain that history has condemned Moldova to be a state. Furthermore, to this debate on national identity, problems of secessionism in the south and east of this state have arised. Language, unification to Romania or not, relationship with Russia, which politics to adopt toward minorities : these are the debates which have structured the political field in the first years of postcommunism in Moldova. Yet, the Romanian's patronizing attitude has pushed the Moldovan leadership into adopting a stronger line on Moldovan identity. This provides the current government a considerable political capital.

\section{AUTEURS}

\section{CHARLES KING}

C. King est chercheur au New College à Oxford. 